\title{
Usefulness of an index score as a predictor of hepatic fibrosis in obese patients undergoing bariatric surgery
}

\author{
R. Díez Rodríguez ${ }^{1}$, M. D. Ballesteros Pomar $^{2}$, A. Calleja Fernández², T. Gónzalez de Francisco ${ }^{3}$ \\ and J. L. Olcoz Goñi ${ }^{1,4}$ \\ Services of ${ }^{1}$ Digestive Diseases, ${ }^{2}$ Endocrinology and Nutrition, and ${ }^{3}$ Surgery. Complejo Asistencial de León. Spain. \\ ${ }^{4}$ CIBERehd Member
}

\begin{abstract}
Objective: to evaluate the usefulness of a non-invasive clinical score to predict liver fibrosis in the steatosis associated with morbid obesity.

Patients and methods: we included 88 patients, who underwent bariatric surgery in the Sanitary Area of León, Spain, and who showed a liver biopsy with steatosis greater than $5 \%$. This is a retrospective study in which the rate of fibrosis is calculated from tests performed during the preoperative period, and is then compared to data from intraoperative hepatic biopsies. The analysis population was grouped according to the presence of advanced fibrosis in the liver biopsy (grade 3-4) or its absence (grade 0-2). The cutoff used for diagnosing advanced fibrosis was 0.676 (high cutoff point), and the cutoff point to exclude advanced fibrosis was -1.455 (low cutoff).

Results: the prevalence of advanced fibrosis in the histological samples was $5.5 \%$, and $65.9 \%$ of patients had no fibrosis. The cutoff for a low negative predictive value was $100 \%$, and sensitivity was $100 \%$. The cutoff point for a high positive predictive value was $1.7 \%$, and specificity was $31.3 \%$.

Conclusions: this scoring system for morbidly obese patients eligible for bariatric surgery allows to identify those without advanced fibrosis, but cannot predict who may have advanced fibrosis
\end{abstract}

Key words: Morbid obesity. Non-alcoholic fatty liver disease. Fibrosis.

Díez Rodríguez R, Ballesteros Pomar MD, Calleja Fernández A, Gónzalez de Francisco T, Olcoz Goñi JL. Usefulness of an index score as a predictor of hepatic fibrosis in obese patients undergoing bariatric surgery. Rev Esp Enferm Dig 2009; 101: 528-535.

Received: 28-01-09

Accepted: 06-05-09.

Correspondence: Rubén Díez Rodríguez. Sección de Aparato Digestivo. Complejo Asistencial de León. C/ Altos de Nava, s/n. 24080 León, Spain. e-mail: rudiro@msn.com

\section{INTRODUCTION}

Obesity in Spain is a health problem with a prevalence around $15 \%$ in the adult population (1). Obesity can cause complications at various levels in the human body, the liver being one of the organs most commonly affected.

The liver is affected in the form of non-alcoholic fatty liver, an entity that includes a spectrum of diseases ranging from simple steatosis to cirrhosis. The prevalence of steatosis and non-alcoholic steatohepatitis in obese patients undergoing bariatric surgery is 91 and $32 \%$, respectively (2).

Milder forms of liver damage may progress to more advanced forms. It has been estimated that approximately $53 \%$ of patients with steatosis remain stable, while $32 \%$ progress towards steatohepatitis, of whom 7-16\% progress to cirrhosis $(3,4)$.

Bariatric surgery may be a treatment for fatty liver disease because it improves insulin resistance, which is a pathogenic factor $(5,6)$. It is indicated in those patients who have a BMI greater than 40 or higher than 35 with comorbidities, according to the consensus of the Spanish Society for the Study of Obesity (SEEDO) (7). Multiple studies with paired liver biopsies (during and after surgery) have shown improved liver histology after weight loss surgery (8-10).

Liver biopsy is the gold standard method for establishing the diagnosis and prognosis of non-alcoholic fatty liver. It is an invasive method with associated morbidity $(0.3 \%)$ and mortality $(0.01 \%)(11)$, and has certain limitations such as intra- and inter-observer variability, and its ability to only analyze a small portion of liver tissue (1:50.000) (12). Because of these limitations liver biopsy is not deemed a diagnostic method applicable to the general population.

Given the potential progression of non-alcoholic fatty liver to advanced fibrosis, a less invasive diagnostic 
method to identify patients with advanced fibrosis earlier, so they are subjected to monitoring and complications of cirrhosis are prevented, has been sought.

In this regard, and given the impossibility of generalizing biopsy as a diagnostic method, various scores have been developed with clinical and/or laboratory data $(13,14)$. Among the scores published in the literature the proposal by Angulo et al. can be highlighted (15). This scoring system was developed and validated in a population with a high prevalence of advanced liver fibrosis, and showed a suitable diagnostic yield.

The aim of this study was to verify the clinical utility of the scoring system proposed by Angulo et al. (15) for the detection of hepatic fibrosis in obese patients with fatty liver who are candidates to bariatric surgery.

\section{PATIENTS AND METHODS}

\section{Patients}

This is a retrospective study conducted in a third-level hospital. Patients eligible for bariatric surgery were selected in an endocrine clinic according to SEEDO consensus criteria (7).

One hundred and twenty-one patients with morbid obesity in the health area of León, operated between July 1999 and August 2007, were selected. Of these 121 patients, 33 with steatosis smaller than $5 \%$ were excluded, so the study was finally conducted on 88 patients (Fig. 1).

Routine lab tests were performed for all patients before surgery, with the purpose of excluding other causes of liver damage (hepatitis B, hepatitis $\mathrm{C}$, iron profile, immune markers). No patients showed pathological alcohol consumption or used potentially hepatotoxic drugs (methotrexate, tretracyclines, high doses of estrogen or steroids). The procedure was carried out by the same surgical team, who performed a Scopinaro's biliopancreatic diversion.

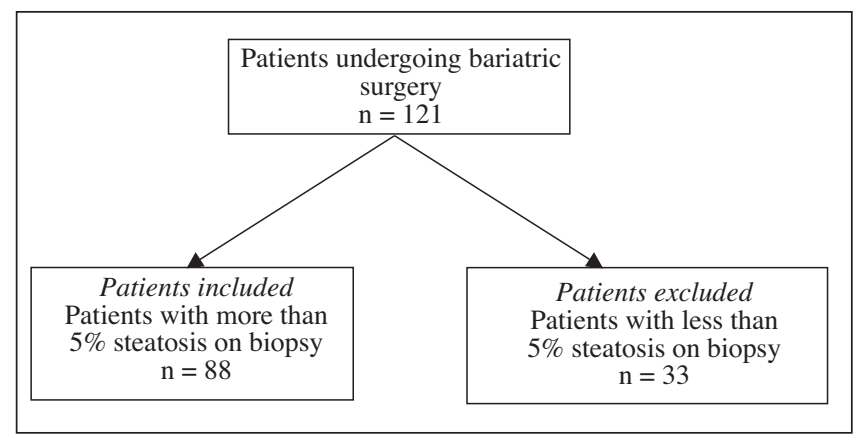

Fig. 1. Classification of patients analyzed in the study. Clasificación de los pacientes analizados en el estudio.

\section{Histological study}

Two biopsies of the right liver lobe using a Tru-cut needle were taken early during the procedure to avoid possible changes in liver histology associated with the intervention. The consent for biopsy was collected with the consent for surgery.

The analysis of the biopsy was performed by a single group of pathologists. Steatosis was classified as minimal (1-5\%), mild (6-33\%), moderate (34-66\%), or severe (67$100 \%)(16)$.

Fibrosis was classified according to the National Institutes of Heath-sponsored NASH Clinical Research Network guidelines: 1) slight fibrosis in zone 3 or portal fibrosis or moderate fibrosis in zone $3 ; 2$ ) fibrosis in zone 3 and the portal area; 3 ) bridging fibrosis; and 4) cirrhosis.

In our study, patients were grouped according to the findings of histology in non-advanced fibrosis $(0-2)$ versus advanced fibrosis (3-4). This division was made with the aim of identifying patients with advanced fibrosis, as these would be those who would benefit from liver biopsy and regular monitoring to prevent cirrhotic complications.

\section{Preoperative assessment}

Laboratory data (fasting glucose, GOT, GPT, albumin, and platelets) were obtained in the preoperative workup.

The criteria of the American Diabetes Association (ADA) were used for the diagnosis of diabetes: two fasting glycemias over $126 \mathrm{mg} / \mathrm{dl}$, or randomly collected glucose levels above $200 \mathrm{mg} / \mathrm{dl}$. Hyperglycemia was diagnosed for patients with a baseline fasting blood glucose greater than $100 \mathrm{mg} / \mathrm{dl}$ (17).

NAFLD fibrosis score was calculated using the formula described by Angulo et al. (15): $0037+-1675 *$ Age (years) $+0.094 *$ BMI $\left(\mathrm{kg} / \mathrm{m}^{2}\right)+1.13 *$ Hyperglycemia or DM (yes $=1$, no $=0)+0.99 *$ GOT / GPT $-0013 *$ Platelets $\left(\mathrm{x} 10^{9} / \mathrm{L}\right)-0.66 *$ Albumin $(\mathrm{g} / \mathrm{dl})$.

\section{Statistical analysis}

Data were collected using Microsoft Excel 2003, and analyzed using SPSS (SPSS, Chicago, IL, USA; V13.0 for Windows). Continuous variables are expressed as mean (standard deviation) values and categorical variables as absolute numbers or percentages. Variables that showed no significance with the Kolmogorov-Smirnov test were considered normal and analyzed for comparisons using Student's t-test for independent variables and ANOVA. Categorical variables were analyzed using the Chi-squared test and Fisher's exact test when necessary. Significance was considered for a $\mathrm{p}$ value $<0.05$.

The calculated values for sensitivity, specificity, and positive and negative predictive values took into account 
the upper and lower cutoffs calculated by Angulo et al. (15). The upper cutoff used for diagnosing advanced fibrosis was 0.676 after applying the formula. The lower cutoff used to exclude advanced fibrosis was a result less than -1.455 after applying the formula.

\section{RESULTS}

\section{Characteristics of the population}

The population included in the study had a mean age of 40.6 years (11.3), and a body mass index (BMI) of $52.7 \mathrm{~kg} / \mathrm{m}^{2}$ (8.2). The mean values of GOT and GPT were 24.3 (14.9) and 34.3 (28.8) units/liter. Average platelet count was $268,000(63,500) \times 10^{\%} / \mathrm{L}$, and albumin 4.1 (0.3) $\mathrm{g} / \mathrm{dl}$. Table I describes the distribution of groups per histological data. The prevalence of advanced fibrosis was $5.5 \%$.

Table I. Distribution of steatosis and fibrosis in the study patients

\begin{tabular}{lc}
\hline Mild steatosis & $51.6 \%$ \\
Moderate steatosis & $33 \%$ \\
Severe steatosis & $15.4 \%$ \\
Without fibrosis & $65.9 \%$ \\
Fibrosis grade 1 & $18.7 \%$ \\
Fibrosis grade 2 & $9.9 \%$ \\
Fibrosis grade 3 & $4.4 \%$ \\
Fibrosis grade 4 & $1.1 \%$ \\
\hline
\end{tabular}

\section{Analysis of fibrosis prediction using the formula and comparing the results with those of liver biopsy}

On applying the formula (with the cutoff points proposed), $42.4 \%$ of patients are in the indeterminate group (the value obtained by applying the formula is between two points).

Patients were divided into two groups, one with advanced fibrosis (grade 3 and 4 ), $n=5$, and one without advanced fibrosis (grade $0-2$ ),$n=83$; values obtained with the formula were compared. Patients with advanced fibrosis had a significantly higher score than patients without advanced fibrosis (fibrosis scores - advanced $v s$. non-advanced, $\mathrm{p}=0.0028$ ). After including the cutoff points proposed by Angulo et al. (15) in a ROC curve analysis, the area under the curve obtained was 0.0776 , revealing a limited diagnostic utility.

On applying the formula, 20 patients (24.09\%) were classified in the group of non-advanced fibrosis, none of these had advanced fibrosis on their biopsy (negative predictive value $100 \%$ and sensitivity 100\%); 58 patients were classified according to the formula in the group of advanced fibrosis, only 1 of these had advanced fibrosis on the biopsy (positive predictive value of $1.7 \%$, specificity $31.3 \%$ ) (Table II).

From a total of 88 patients, $21(23.9 \%)$ were correctly classified. We analyzed the diagnostic performance of other cutoffs, and none of them showed better results than those reported by Angulo et al. (15).

\section{DISCUSSION}

This study has several limitations. First, the study was based on a population with a particular age and body mass index, so the results cannot be extrapolated to the general obese population. Second, the index was developed on a population with features that do not match those of the population in this study. Third, although it was used for histological classification criteria by the same group of pathologists, there is probably some degree of intra- and inter-observer variability in sample classification.

Due to the high prevalence of non-alcoholic fatty liver, many scores are designed to improve the diagnosis of fibrosis. Many of them include parameters not available in clinical laboratories (aminoterminal propeptide of type III collagen, or tissue inhibitor of metalloproteinase 1 , hyaluronic acid, endothelin 1, etc.) $(13,18,19)$, which limits their use in clinical practice. With our improved understanding of the pathogenesis of the disease apoptosis markers have been proposed (cytokeratin 18 as a result of caspase 3 ) to play a role in the differential diagnosis between steatohepatitis and non-alcoholic steatosis (20).

Table II. Comparison of the results after applying the formula to patients in Hospital de León, with the results obtained by Angulo et al. (15) (in the group of patients used for design and validation) and Qureshi (22)

\begin{tabular}{|c|c|c|c|c|c|}
\hline & & Qureshi (22) & $\begin{array}{c}\text { Angulo: } \\
\text { estimation group } \\
\text { (15) }\end{array}$ & $\begin{array}{c}\text { Angulo: } \\
\text { validation group } \\
\text { (15) }\end{array}$ & Hospital of León \\
\hline High cutoff & $\begin{array}{c}\text { NPV } \\
\text { Sensitivity }\end{array}$ & $\begin{array}{l}98 \% \\
96 \%\end{array}$ & $\begin{array}{l}93 \% \\
82 \%\end{array}$ & $\begin{array}{l}88 \% \\
77 \%\end{array}$ & $\begin{array}{l}100 \% \\
100 \%\end{array}$ \\
\hline Low cutoff & $\begin{array}{c}\text { PPV } \\
\text { Specificity }\end{array}$ & $\begin{array}{l}33 \% \\
84 \%\end{array}$ & $\begin{array}{l}90 \% \\
98 \%\end{array}$ & $\begin{array}{l}82 \% \\
96 \%\end{array}$ & $\begin{array}{c}1.7 \% \\
31.3 \%\end{array}$ \\
\hline
\end{tabular}


Fibroscan has been recently introduced as a non-invasive method for diagnosing liver fibrosis in patients with non-alcoholic fatty liver (21). The results are encouraging because they get to a level of F3 and F4 fibrosis, an area under the curve increased (0.9). More studies are needed to validate its usefulness in patients with non-alcoholic fatty liver disease.

The system proposed by Angulo et al. (15) is a noninvasive method for the diagnosis of fibrosis. The parameters included in the formula are routinely analyzed in routine clinical practice. It was designed by a multicenter study in 480 patients, and was validated on 253. In total, $27 \%$ of patients in this study showed advanced fibrosis, while $33.3 \%$ did not show fibrosis.

This score was applied afterwards by Qureshi et al. (22) on 331 morbid obesity subjects who underwent bariatric surgery; $36.5 \%$ of patients had no fibrosis, and $13.6 \%$ had advanced fibrosis. Unlike the series, the results presented here show $65.9 \%$ of patients suffering from fibrosis, while only $5.5 \%$ showed advanced fibrosis in the study (Table I).

Furthermore, the index was designed in a population with a mean age of 47.7 years and a mean BMI of 32.42 $\left(\mathrm{kg} / \mathrm{m}^{2}\right)$, while the average age of patients in our study was 41.2 and BMI was $52.7\left(\mathrm{~kg} / \mathrm{m}^{2}\right)$. Qureshi et al. (22) show a mean age of 40.5 and BMI of $48.4\left(\mathrm{~kg} / \mathrm{m}^{2}\right)$. The different values of BMI, although part of a formula, might have influenced validity in our group.

The data for the low cutoff are consistent with data published to date. In addition to the high cutoff point, the positive predictive value is very low when compared to other studies (Table II). The different values observed are probably due to a low prevalence of advanced fibrosis, since patients have different characteristics. As BMI increases in the population, a decrease in PPV and specificity for high cutoffs occurs.

The study published by Angulo et al. (15), for an estimated 5\% prevalence of advanced fibrosis, found a positive predictive value for the high cutoff point of $64 \%$ (95\% CI 53-75). A value much higher than that was obtained in this study. No other cutoffs had a better performance than that proposed by Angulo et al. (15).

On applying this index many patients are classified as indeterminate $(42.4 \%)$, and only a small fraction of obese patients will benefit from this index. It cannot be assumed that patients with a score above the high cutoff point had advanced fibrosis.

It seems reasonable to apply this index to morbidly obese patients, given its ease of implementation, and given that those below the low cutoff will likely have no fibrosis (NPV 100\%). A total of $22.7 \%$ of our population will benefit from this index.

More studies will be needed to validate this index in various populations of obese patients, and perhaps to change some of the parameters in the formula according to the different populations in order to improve its utility.

\section{REFERENCES}

1. Aranceta-Bartrina J, Serra-Majem L, Foz-Sala M, Moreno-Esteban B. Prevalence of obesity in Spain. Med Clin (Barc) 2005; 125(12): 460-6.

2. Machado M, Marques-Vidal P, Cortez-Pinto H. Hepatic histology in obese patients undergoing bariatric surgery. J Hepatol 2006; 45(4): 600-6.

3. Buque X, Aspichueta P, Ochoa B. Molecular basis of obesity-related hepatic steatosis. Rev Esp Enferm Dig 2008; 100(9): 565-78.

4. Ratziu V, Giral P, Charlotte F, Bruckert E, Thibault V, Theodorou I, et al. Liver fibrosis in overweight patients. Gastroenterology 2000; 118(6): 1117-23.

5. Solís-Herruzo JA, Solís-Muñoz P. Genetic factors in non-alcoholic fatty liver disease. Rev Esp Enferm Dig 2008; 100(4): 195-201.

6. Solís Herruzo JA, García Ruiz I, Pérez Carreras M, Muñoz Yagüe MT. Non-alcoholic fatty liver disease. From insulin resistance to mitochondrial dysfunction. Rev Esp Enferm Dig 2006; 98(11): 844-74.

7. Salas-Salvado J, Rubio MA, Barbany M, Moreno B. SEEDO 2007 Consensus for the evaluation of overweight and obesity and the establishment of therapeutic intervention criteria. Med Clin (Barc) 2007; 128(5): 184-96.

8. Kral JG, Thung SN, Biron S, Hould FS, Lebel S, Marceau S, et al. Effects of surgical treatment of the metabolic syndrome on liver fibrosis and cirrhosis. Surgery 2004; 135(1): 48-58.

9. Liu X, Lazenby AJ, Clements RH, Jhala N, Abrams GA. Resolution of nonalcoholic steatohepatits after gastric bypass surgery. Obesity Surgery 2007; 17(4): 486-92.

10. Silverman EM, Sapala JA, Appelman HD. Regression of hepatic steatosis in morbidly obese persons after gastric bypass. Am J Clin Pathol 1995; 104(1): 23-31.

11. Piccinino F, Sagnelli E, Pasquale G, Giusti G. Complications following percutaneous liver biopsy. A multicentre retrospective study on 68,276 biopsies. J Hepatol 1986; 2(2): 165-73.

12. Adams LA, Angulo P. Role of liver biopsy and serum markers of liver fibrosis in non-alcoholic fatty liver disease. Clin Liver Dis 2007; 11(1): 25-35.

13. Rosenberg WM, Voelker M, Thiel R, Becka M, Burt A, Schuppan D, et al. Serum markers detect the presence of liver fibrosis: a cohort study. Gastroenterology 2004; 127(6): 1704-13.

14. Ratziu V, Massard J, Charlotte F, Messous D, Imbert-Bismut F, Bonyhay L, et al. Diagnostic value of biochemical markers (FibroTest-FibroSURE) for the prediction of liver fibrosis in patients with non-alcoholic fatty liver disease. BMC Gastroenterology 2006; 6: 6 .

15. Angulo P, Hui JM, Marchesini G, Bugianesi E, George J, Farrell GC, et al. The NAFLD fibrosis score: a noninvasive system that identifies liver fibrosis in patients with NAFLD. Hepatology 2007; 45(4): 84654

16. Brunt EM. Pathology of fatty liver disease. Mod Pathol 2007; 20(Supl. 1): S40-8.

17. Association AD. Diagnosis and classification of diabetes mellitus. Diabetes Care. 2008; 31(Supl. 1): S55-60.

18. Degertekin B, Ozenirler S, Elbeg S, Akyol G. The serum endothelin1 level in steatosis and NASH, and its relation with severity of liver fibrosis. Dig Dis Sci 2007; 52(10): 2622-8.

19. Suzuki A, Angulo P, Lymp J, Li D, Satomura S, Lindor K. Hyaluronic acid, an accurate serum marker for severe hepatic fibrosis in patients with non-alcoholic fatty liver disease. Liver Int 2005; 25(4): 779-86.

20. Wieckowska A, Zein NN, Yerian LM, López AR, McCullough AJ, Feldstein AE. In vivo assessment of liver cell apoptosis as a novel biomarker of disease severity in nonalcoholic fatty liver disease. Hepatology 2006; 44(1): 27-33.

21. Yoneda M, Mawatari H, Fujita K, Endo H, Iida H, Nozaki Y, et al. Noninvasive assessment of liver fibrosis by measurement of stiffness in patients with nonalcoholic fatty liver disease (NAFLD). Dig Liver Dis 2008; 40(5): 371-8.

22. Qureshi K, Clements RH, Abrams GA. The utility of the "NAFLD fibrosis score" in morbidly obese subjects with NAFLD. Obes Surg 2008; 18(3): 264-70 